\title{
Activation of FXR pathway does not alter glial cell function
}

Stefanie Albrecht ${ }^{1 \dagger}$, Ann-Katrin Fleck ${ }^{2 \dagger}$, Ina Kirchberg ${ }^{1}$, Stephanie Hucke ${ }^{2}$, Marie Liebmann ${ }^{2}$, Luisa Klotz ${ }^{2^{*+}}$ and Tanja Kuhlmann ${ }^{1 * \dagger}$

\begin{abstract}
Background: The nuclear receptor farnesoid-X-receptor (FXR; NR1H4) is expressed not only in the liver, gut, kidney and adipose tissue but also in the immune cells. FXR has been shown to confer protection in several animal models of inflammation, including experimental autoimmune encephalomyelitis (EAE), an animal model of multiple sclerosis (MS). FXR agonists are currently tested in clinical trials for treatment of human metabolic diseases. The beneficial effect of FXR agonists in EAE suggests that FXR might represent a potential target in inflammatory-demyelinating CNS diseases, such as MS. In MS, oligodendrocytes not only undergo cell death but also contribute to remyelination. This repair mechanism is impaired due to a differentiation block of oligodendroglial progenitor cells. Activation of other nuclear receptors that heterodimerize with FXR promote oligodendroglial differentiation. Therefore, we wanted to address the functional relevance of FXR for glial cells, especially for oligodendroglial differentiation.

Methods: We isolated primary murine oligodendrocytes from FXR-deficient (FXR Ko) and wild-type (WT) mice and determined the effect of FXR deficiency and activation on oligodendroglial differentiation by analysing markers of oligodendroglial progenitor cells (OPCs) and mature oligodendrocytes (OLs) using qRT-PCR and immunocytochemistry. Additionally, we determined whether FXR activation modulates the pro-inflammatory profile of astrocytes or microglia and whether this may subsequently modulate oligodendroglial differentiation. These in vitro studies were complemented by histological analyses of oligodendrocytes in FXR Ko mice.
\end{abstract}

Results: FXR is expressed by OPCs and mature oligodendrocytes. However, lack of FXR did not affect oligodendroglial differentiation in vitro or in vivo. Furthermore, activation of FXR using the synthetic agonist GW4064 did not affect oligodendroglial differentiation, remyelination in an ex vivo model or the expression of pro-inflammatory molecules in astrocytes or microglia. Concordantly, no effects of supernatants from macrophages cultured in the presence of GW4064 were observed regarding a possible indirect impact on oligodendroglial differentiation.

Conclusions: Our data suggest that FXR is dispensable for oligodendroglial differentiation and that FXR agonists, such as GW4064, represent a potential therapeutic approach for MS which specifically targets peripheral immune cells including macrophages but not brain-resident cells, such as oligodendrocytes, astrocytes or microglia.

Keywords: FXR, Nuclear receptors, Oligodendrocytes, Microglia, Astrocytes, GW4064

\footnotetext{
* Correspondence: luisa.klotz@ukmuenster.de;

tanja.kuhlmann@ukmuenster.de

Stefanie Albrecht and Ann-Katrin Fleck are co-first authors who equally

contributed to this paper.

Luisa Klotz and Tanja Kuhlmann are co-last authors who equally contributed

to this paper.

${ }^{\dagger}$ Equal contributors

${ }^{2}$ Department of Neurology, University of Münster, 48149 Münster, Germany

${ }^{1}$ Institute of Neuropathology, University Hospital Münster, 48149 Münster,

Germany
}

(c) The Author(s). 2017 Open Access This article is distributed under the terms of the Creative Commons Attribution 4.0 International License (http://creativecommons.org/licenses/by/4.0/), which permits unrestricted use, distribution, and reproduction in any medium, provided you give appropriate credit to the original author(s) and the source, provide a link to the Creative Commons license, and indicate if changes were made. The Creative Commons Public Domain Dedication waiver (http://creativecommons.org/publicdomain/zero/1.0/) applies to the data made available in this article, unless otherwise stated. 


\section{Background}

Nuclear receptors are a large group of transcription factors that comprises 49 family members which enable the organism to quickly adapt to environmental changes by inducing the appropriate gene program [1]. Nuclear receptors are activated by lipophilic ligands including hormones, fatty acids, bile acids and oxysterols, homo- and heterodimerize upon activation and regulate the metabolism of glucose, fatty acids, triglycerides and lipoproteins [1]. Besides these metabolic effects, numerous immune-modulatory effects by nuclear receptors have been described, including modulation of central nervous system (CNS) autoimmunity [2-4].

Interestingly, several members of the nuclear receptor family are also involved in molecular mechanisms regulating oligodendroglial differentiation. Downregulation of retinoid X receptor gamma (RXRY) expression or application of $\mathrm{RXR} \gamma$ antagonists significantly impaired oligodendroglial differentiation. Similarly, mice lacking $\mathrm{RXR} \gamma$ showed delayed remyelination after induction of demyelination in vivo [5]. In oligodendrocytes, RXR $\gamma$ heterodimerizes with the vitamin $\mathrm{D}$ receptor (VDR) to induce oligodendroglial differentiation, and accordingly, vitamin $\mathrm{D}$-induced activation accelerates oligodendroglial differentiation [6]. Other nuclear receptors, such as thyroid receptors (TR) and liver X receptors (LXR), are well expressed in oligodendroglial lineage cells and modulate oligodendroglial differentiation and myelination. Patients with congenital hypoparathyroidism typically develop a hypomyelinating phenotype, and triiodothyronine (T3) is required for the maturation of oligodendroglial progenitor cells into mature myelinating oligodendrocytes (for review see [7]). Mice lacking both LXR $\alpha$ and LXR $\beta$ display thinner myelin sheaths and reduced expression levels of myelin genes in the cerebellum. Furthermore, addition of pharmacological activators of LXRs to mixed glial cell cultures promotes oligodendroglial differentiation [8].

The nuclear receptor farnesoid-X-receptor (FXR; NR1H4) is expressed not only in the liver, gut, kidney and adipose tissue, but also in the immune cells $[9,10]$. FXR heterodimerizes with other nuclear receptors, such as RXRs [11, 12]. The best-characterized endogenous FXR ligands are lipophilic bile acids, namely cholic acid and chenodeoxycholic acid. In addition, the synthetic compound GW4064 serves as an agonist with high receptor specificity and efficacy $[11,13,14]$. In accordance with its expression pattern, FXR activation has been shown to confer protection in several animal models of innate intestinal and hepatic inflammation [11]. In experimental autoimmune encephalomyelitis (EAE), an animal model of multiple sclerosis (MS), pharmacological activation of FXR significantly ameliorated the disease course by induction of anti-inflammatory macrophages $[15,25]$. Interestingly, FXR agonists are currently tested in clinical trials for treatment of alcoholic hepatitis, type 2 diabetes mellitus and primary biliary cirrhosis, demonstrating that FXR already represents an attractive pharmacological target in human metabolic diseases. The beneficial effect of FXR agonists in EAE suggests that FXR might represent a potential target in inflammatory-demyelinating CNS diseases, such as MS. Oligodendroglial lineage cells are not only a primary target in MS but also a prerequisite for remyelination, an endogenous repair mechanism. However, especially in chronic MS lesions, remyelination is limited at least partly due to a differentiation block inhibiting the differentiation of oligodendroglial progenitor cells (OPCs) into myelinating oligodendrocytes [16-18]. Importantly, remyelination failure contributes to axonal damage and worsening of clinical signs in demyelinating animal models $[19,20]$.

Given its recently illustrated relevance in macrophagemediated protection from CNS autoimmunity and the functional role of several nuclear receptors for oligodendroglial differentiation, we addressed the functional relevance of FXR in different brain-resident cells, especially focusing on oligodendrocytes. Here, we show that FXR is expressed in murine oligodendrocytes. However, lack or activation of FXR did not influence oligodendroglial differentiation in vitro or in vivo. Furthermore, FXR activation did not affect key immune functions of astrocytes and microglial cells. These data demonstrate that, in contrast to other nuclear receptors, FXR is dispensable for oligodendroglial differentiation as well as for brainresident astrocytes and microglia and hence suggest that pharmacological activation of FXR to downregulate CNS inflammation does not interfere with repair mechanisms, such as remyelination.

\section{Materials and methods \\ Animals}

FXR Ko mice [21] were purchased from Jackson Laboratory and $\mathrm{C} 57 \mathrm{Bl} / 6$ wild-type control mice from the animal facility in Münster, Germany. Animal handling and experiments were conducted according to the German Animal Welfare Act and approved by the responsible governmental authorities (LANUV Nordrhein-Westfalen; AZ-8.84.02.05.20.12.286, 84.-02.04.2013.A029, AZ 84-02.04.2014. A221, AZ 84-02.04.2013.A374).

\section{Primary oligodendroglial culture and treatment}

Primary OPCs were isolated using the immunopanning method as described earlier [22, 23]. OPCs/differentiating oligodendrocytes were incubated with 1 or $10 \mu \mathrm{M}$ GW4064 (Tocris, 2473) for 24/48 h. Cell viability upon treatment was measured according to manufacturer's protocol using CellTiter-Glo ${ }^{\circ}$ Luminescent Cell Viability Assay (Promega, G7570). When treated with bone 
marrow-derived macrophage (BMM) supernatant, oligodendrocytes were incubated with $50 \%$ supernatant in culture medium for $48 \mathrm{~h}$.

\section{Cerebellar slice cultures and treatment}

Sagittal, cerebellar slices from P1 mice were dissected as described earlier [23]. After 12 days in culture, demyelination was induced by $16-\mathrm{h}$ incubation with $0.5 \mathrm{mg} / \mathrm{ml} \mathrm{ly-}$ solecithin. During subsequent remyelination, the brain slices were incubated with 10 or $20 \mu \mathrm{M}$ GW4064 for 14 days. The ex vivo slices were fixed and stained with anti-neurofilament $70 \mathrm{kDa}$ (NFL; Dako, 1:500) and antiMBP (Abcam, ab7349, 1:200). Confocal $z$-stacks of whole slices were acquired with a Zeiss LSM 700 confocal microscope, and images were analysed using NIH ImageJ. The extent of myelination was quantified by determining the ratio between MBP and NFL staining. Two independent experiments were performed, and in each experiment, six brain slices and three z-stacks from six brain slices were analysed for each concentration of GW4064 or DMSO.

\section{Macrophage and microglia culture and treatment}

Preparation of bone marrow-derived macrophages and generation of primary astrocyte cultures via mixed glial culture preparation were performed as described previously [2]. The murine embryonic stem cell-derived microglial precursor cells (ESdMs) were provided by Harald Neumann (University of Bonn) and cultured according to their publication $[2,24]$. Depending on the individual cell type, cells were treated with different concentrations of the synthetic FXR agonist GW4064 (Tocris, 2473); concentrations depended on individual cell type. Control groups were treated with the equivalent volume of solvent, i.e. DMSO. ESdMs and primary astrocytes were incubated for $24 \mathrm{~h}$ with the BMM-conditioned supernatant, before stimulation with LPS and IFN $\gamma$.

\section{Generation of BMM supernatant}

To produce BMM-conditioned supernatant, BMMs were treated with $15 \mu \mathrm{M}$ GW4064 or DMSO and cultured for $72 \mathrm{~h}[25]$.

\section{Detection of TNFa and nitric oxide}

TNF $\alpha$ in cell culture supernatants was quantified using ELISA Ready-SET-Go!" (murine TNF $\alpha$; eBioscience) according to the manufacturer's recommendations. Nitrite concentrations were measured in the supernatants using the Griess Reagent Kit (Invitrogen) according to the manufacturer's instructions.

\section{$\mathrm{RT}^{2}$ Profiler $^{\mathrm{TM}} \mathrm{PCR}$ Array}

Treated ESdMs and astrocytes were stimulated with LPS and INFy for $6 \mathrm{~h}$. RNA isolation was performed with RNeasy Mini Kit (Qiagen) combined with the RNase-
Free DNase Set (Qiagen) to digest DNA. For transcription to complementary DNA (cDNA), the $\mathrm{RT}^{2}$ FirstStrand Kit (Qiagen) was utilized. Afterwards, the $\mathrm{RT}^{2}$ Profiler $^{\mathrm{Tm}}$ PCR Array Inflammatory Response \& Autoimmunity (\#PAMM-077Z; Qiagen) was performed with $\mathrm{RT}^{2}$ Real-Time SYBR Green PCR Master Mix (SuperArray Bioscience) according to the manufacturer's protocol. For normalization, the housekeeping gene Hsp90ab1 was taken as internal control. Analysis was performed at the $\mathrm{RT}^{2}$ Profiler PCR Array Data Analysis Web portal (provided from Qiagen).

\section{RNA isolation and qRT-PCR}

Total RNA from cells was isolated using peqGOLD Total RNA Kit (12-6634, PeqLab Biotechnologie GmbH). Messenger RNA (mRNA) was transcribed into cDNA by reverse transcription reaction (High Capacity cDNA Transcription Kit, Applied Biosystems), and cDNA was diluted to a final concentration of $0.75 \mathrm{ng} / \mu \mathrm{l}$. qRT-PCR was performed using Power SYBR ${ }^{\circ}$ Green PCR Master Mix (Applied Biosystems) and StepOne Plus real-time cycler (Applied Biosystems). The following primers were used: Fxr fw: GCCACAGATTTCCTCCTCGT, $F x r$ rev: CAGT CTCTCCCTGGTACCCA, $M b p$ fw: GTACAAGGACTC ACACACGAGA, $M b p$ rev: GTTCGAGGTGTCACAA TGTTCT, RPLPO fw: CGACCTGGAAGTCCAACTAC and RPLPO rev: ATCTGCTGCATCTGCTTG, and data were normalized using $R P L P O$ as internal control.

\section{Immunocytochemistry (ICC)}

OPCs were fixed directly after seeding or differentiated and fixed after $48 \mathrm{~h}$ as mature oligodendrocytes. Cells were permeabilised for $10 \mathrm{~min}$ in $0.5 \%$ Triton X-100 in PBS and blocked using 5\% FCS/PBS for $30 \mathrm{~min}$. The primary antibodies were rat anti-MBP (Abcam, ab7349, 1:200), rabbit anti-PDGFR $\alpha$ (Santa Cruz, SSC338, 1:300), rabbit-FXR NR1H4 (Abcam, ab28676, 1:200; or Santa Cruz sc-13063, 1:100) and mouse anti-Olig2 (Medac, $387 \mathrm{M}-16,1: 200)$. Incubation was performed at $4{ }^{\circ} \mathrm{C}$ over night. Secondary antibody staining was performed using $\mathrm{Cy}^{\mathrm{rm}} 3$ Anti-Rat (1:500) (Jackson, 112-165-167) and antiRabbit Alexa Fluor 488 conjugate (1:500) (Jackson, 111545-144) for $2 \mathrm{~h}$ at RT before embedding with RotiMount FluorCare DAPI (Carl Roth, HP20.1). Images were taken using the laser scanning microscope (LSM 700, Zeiss Jena). At least 200 cells were quantified, and the numbers of MBP+ and PDGFR $\alpha+$ cells were assessed as percentage of total DAPI+ cells.

\section{Immunohistochemistry (IHC)}

Ten-day and 8-week-old WT or FXR Ko mice were sacrificed and intracardially perfused. The spleens, spinal cords and brains were removed and fixed in 4\% PFA overnight. Paraffin sections $(4 \mu \mathrm{m})$ were pretreated with 
citrate buffer $(\mathrm{pH}$ 6) and stained using an automated immunostainer (AutostainerLink 48, Dako). Primary antibodies were specific to FXR (NR1H4, rabbit, Abcam ab28676 1:200; or Santa Cruz sc-13063, 1:50), NogoA (mouse, 11c7, a generous gift from M.E. Schwab, Brain Research Institute, University of Zürich and Department of Biology, Swiss Federal Institute of Technology Zürich, Switzerland, 1:15,000) and Olig2 (rabbit, 18953, IBL, 1:150 and mouse, 387M-16, Medac, 1:200). Numbers of oligodendroglial cells were quantified in a blinded fashion in the corpus callosum, cerebellum and spinal cord in standardized microscopic fields of $10,000 \mu \mathrm{m}^{2}$ each defined by an ocular morphometric grid.

\section{Statistics}

All cell culture experiments were performed in triplicates and replicated at least three times. All statistical analyses were performed using GraphPad Prism 5.03 (GraphPad Software Inc., San Diego, CA). In text and figures, results are provided as mean \pm SEM. Multiple comparisons in the same data set were analysed by the Bonferroni-corrected (for selected groups) one-way ANOVA tests. Single comparisons to control were made using two-tailed Student's $t$ test. $p$ values $<0.05$ were considered significant.

\section{Results}

\section{FXR is expressed in oligodendrocytes}

Histological analysis of brain sections from adult mice revealed expression of FXR in oligodendroglial lineage cells, which were identified by their typical linear arrangement within the corpus callosum (Fig. 1a (arrows)) and by coexpression with OLIG2 (Fig. 1b). Additionally, we analysed expression of FXR in oligodendrocytes in vitro. Before induction of differentiation using CNTF and NT3 [22], almost $100 \%$ of the oligodendroglial lineage cells express PDGFR $\alpha$, a marker specifically expressed by OPCs. After $24 \mathrm{~h}$ of differentiation, approximately $15 \%$ of the cells express MBP, a marker of mature oligodendrocytes; this percentage increases to $35 \%$ after $48 \mathrm{~h}$, whereas PDGFR $\alpha+$ OPCs account for approximately $10 \%$ of the cells. FXR protein expression was detected in OPCs and mature oligodendrocytes using immunocytochemistry (Fig. 1c). Quantification of Fxr gene expression demonstrated an increase in Fxr
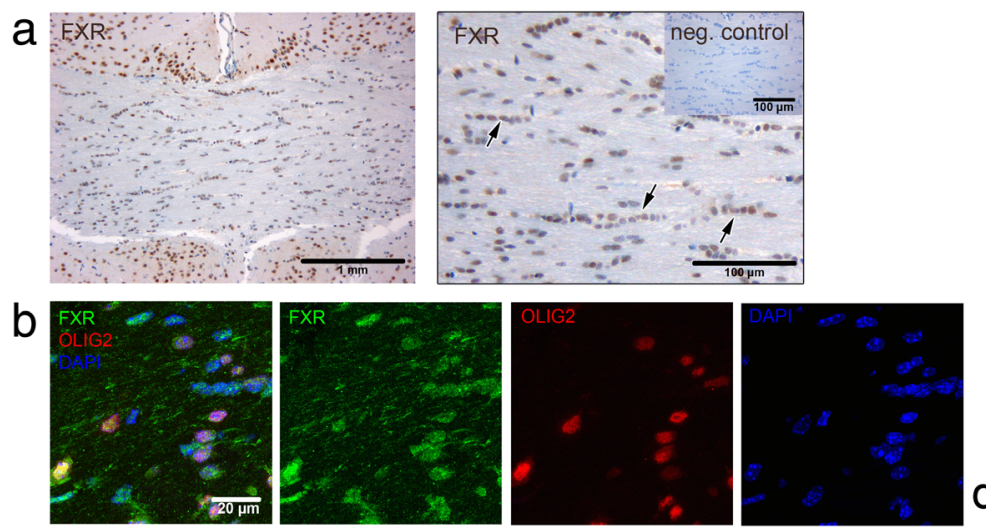

C
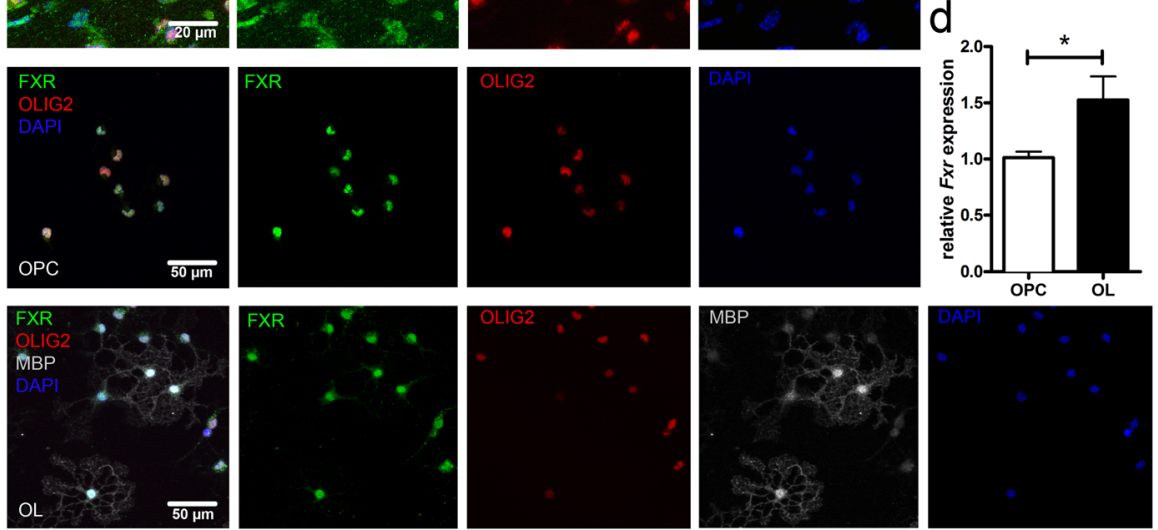

Fig. 1 FXR is expressed by oligodendroglial cells in vivo and in vitro. Immunohistochemical analysis revealed oligodendroglial cells in the corpus callosum of adult mice express FXR protein. Oligodendrocytes were identified by their linear arrangement (arrows) and coexpression of OLIG2, respectively $(\mathbf{a}, \mathbf{b})$. In primary, murine oligodendroglial cells, FXR is expressed in OLIG2+ OPCs and mature oligodendrocytes, identified by OLIG2 and MBP expression after $48 \mathrm{~h}$ of in vitro differentiation (c). The relative Fxr gene expression level is increased in mature oligodendrocytes compared to OPC (d), $n=3$; two-tailed Student's $t$ test, ${ }^{*} p<0.05$; all images are representative 
mRNA expression during oligodendroglial differentiation (Fig. 1d).

\section{Lack of FXR does not impair oligodendroglial differentiation in vivo or in vitro}

Utilizing FXR Ko mice, we determined the impact of FXR loss on the numbers of oligodendrocytes 10 days and 8 weeks after birth. To determine the numbers of OPCs and mature oligodendrocytes, we used OLIG2, a transcription factor that is expressed in both cell populations. Quantification of OLIG2+ cells in the corpus callosum, cerebellum and spinal cord of FXR Ko and WT mice did not reveal differences between the two genotypes (Fig. 2a, c). Additionally, the number of mature NogoA positive oligodendrocytes was not affected by loss of FXR in any of the analysed CNS regions (Fig. 2b, d). The differentiation of FXR Ko or WT oligodendrocytes isolated from either cerebrum or cerebellum was examined in vitro (Fig. 2e-h). After $24 \mathrm{~h}$ of differentiation, $M b p$ expression levels were slightly reduced in FXR Ko cells; however, no significant alteration in gene expression could be observed in either cerebral or cerebellar oligodendrocytes (Fig. 2e, g). In addition, no difference in the amount of PDGFR $\alpha+$ OPCs or $\mathrm{MBP}+$ mature oligodendrocytes could be detected comparing FXR Ko and WT cells (Fig. 2f, h). In summary, lack of FXR had no major effect on the numbers of oligodendrocytes or oligodendroglial differentiation, neither in vivo nor in vitro.

\section{Activation of FXR does not affect oligodendroglial differentiation}

OPCs isolated either from the cerebrum or from the cerebellum were treated with 1 or $10 \mu \mathrm{M}$ of the pharmacological FXR agonist GW4064 after induction of differentiation for up to $48 \mathrm{~h}$. GW4064 treatment did not affect the viability of the oligodendroglial cells (data not shown). mRNA expression levels of the myelin-associated gene $M b p$ increased over time, but no differences in the relative expression levels were detected between GW4064treated and control-treated oligodendrocytes (Fig. 3a). Using immunocytochemistry, we determined the number of PDGFR $\alpha+$ OPCs and MBP+ mature oligodendrocytes after $48 \mathrm{~h}$ of GW4064 treatment. The addition of

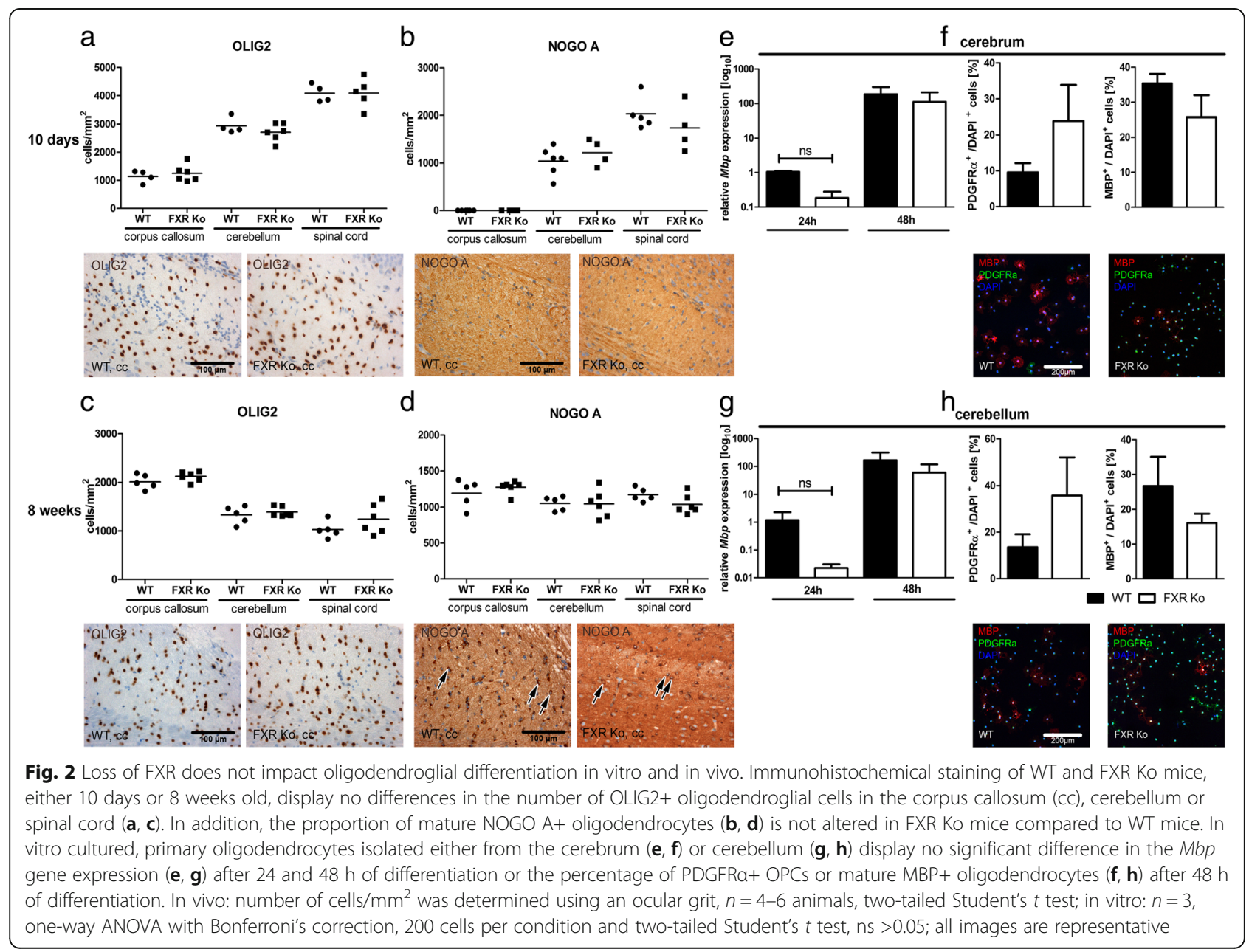




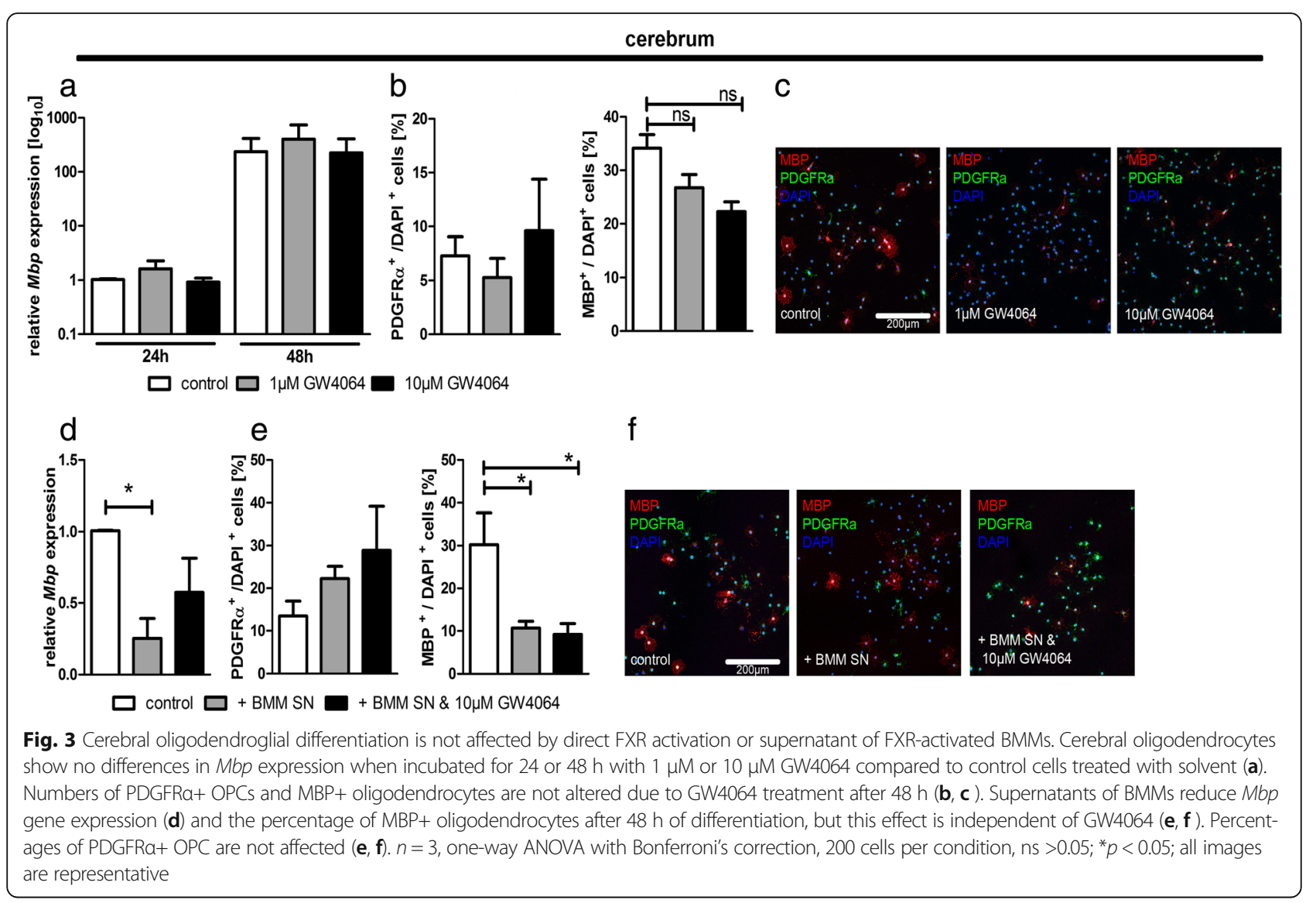

GW4064 to oligodendroglial cell cultures did not result in a significant change in the percentage of PDGFR $\alpha+$ OPCs or MBP+ mature oligodendrocytes (Fig. 3b, c). Additionally, we analysed the impact of GW4064 application to oligodendroglial cells isolated from the cerebellum; however, no alterations in $M b p$ gene expression or changes in the percentage of PDGFR $\alpha+$ OPCs and $\mathrm{MBP}+$ mature oligodendrocytes compared to controls were observed (Additional file 1: Figure S1a-c). These data show that direct activation of FXR has no impact on oligodendroglial differentiation.

\section{Remyelination following toxic-induced demyelination in cerebellar brain cultures is not impacted by activation of FXR}

To analyse a potential impact of FXR activation on the process of remyelination, we made use of cerebellar slice cultures that were demyelinated by lysolecithin. We applied two different concentrations of GW4064 to demyelinated cerebellar slice cultures during 14 days of remyelination. Evaluation of the amount of $\mathrm{MBP}+$ and NFL+ axons revealed no influence of GW4064 treatment on the remyeliation capacity of oligodendrocytes after toxic demyelination in this ex vivo model (Additional file 1: Figure S1g, h).
No indirect effect of FXR-activated macrophages on oligodendroglial differentiation

Since peripheral macrophages migrate into the CNS during MS pathogenesis and then modulate the inflammatory activity of brain-resident cells and given the strong immune-modulatory properties of FXR in macrophages [25], we next addressed whether FXR might indirectly affect oligodendrocytes via FXR-modulated macrophages. To this end, bone marrow-derived macrophages (BMM) were cultured and treated with $10 \mu \mathrm{M}$ GW4064 to activate FXR [25]. Subsequently, OPCs isolated from the cerebrum were cultured in the presence of supernatants from GW4064-exposed BMMs to analyse the effect on oligodendroglial differentiation. Controls included oligodendroglial lineage cells cultured either in the presence of supernatants from BMM without GW4064 exposure or without any BMM supernatants. After $48 \mathrm{~h}$ of differentiation, $M b p$ expression levels and numbers of mature $\mathrm{MBP}+$ oligodendrocytes were significantly reduced in cerebral oligodendrocytes exposed to BMM supernatants; however, this was independent of GW4064 treatment (Fig. 3d-f). No significant differences in the numbers of PDGFR $\alpha+$ OPCs were observed in cultures exposed to BMM-conditioned medium with or without GW4064 or controls (Fig. 3e, f). Cerebellar 
oligodendrocytes showed no significant alteration of $M b p$ gene expression, but a significant decrease in $\mathrm{MBP}+$ mature oligodendrocytes after addition of BMM supernatants compared to controls without BMM supernatants; however, conditioning with GW4064 had no additional effect on oligodendroglial differentiation (Additional file 1: Figure S1d-f). Furthermore, no significant differences in the numbers of PDGFR $\alpha+$ OPCs were observed (Additional file 1: Figure S1e, f).

FXR activation does not affect immune functions of brainresident astrocytes and microglia

In light of the described anti-inflammatory properties of FXR on macrophages [25], we wondered whether pharmacological FXR activation might suppress inflammatory activity of brain-resident astrocytes or microglia, which could also indirectly influence oligodendrocyte differentiation and function in local inflammatory processes during CNS autoimmunity. However, FXR activation in primary astrocytes by different concentrations of GW4064 did not affect the production of key proinflammatory molecules TNF $\alpha$ (Fig. 4a) and NO (Fig. 4b). In this line, also activation of FXR in embryonic stem cell-derived microglial cells (ESdMs; [24]) did not suppress TNF $\alpha$ (Fig. 4g) and NO (Fig. 4h) production. Furthermore, no differences in the expression of 84 genes involved in inflammatory immune responses, such as genes of cytokines, interleukins and chemokines, were observed in DMSO-treated cells compared to GW4064treated astrocytes (Fig. 4c, d) and ESdMs (Fig. 4i, j), respectively.

\section{Supernatant of GW4064-treated BMMs does not affect astrocytic and microglial immune functions}

We next investigated the influence of conditioned supernatant from FXR-activated BMMs on astrocytes and microglial cells. However, there was no modulation of TNF $\alpha$ (Fig. 4e, k) or NO (Fig. 4f, l) production detectable in astrocytes as well as in microglial cells upon incubation with conditioned supernatants. In conclusion, these data indicate that FXR does not play a major role in controlling pro-inflammatory activities of brain-resident cells, neither directly nor indirectly by modulating inflammatory macrophage activity.

\section{Discussion}

Here, we demonstrate that FXR is expressed by OPCs and mature oligodendrocytes, like microglia and astrocytes [25]. However, lack of FXR did not affect oligodendroglial differentiation in vitro or in vivo. Furthermore, activation of FXR using the synthetic agonist GW4064 did not affect oligodendroglial differentiation, ex vivo remyelination or pro-inflammatory activation of astrocytes or microglia.
Several nuclear receptors, such as RXR $\alpha, \operatorname{RXR} \beta, \mathrm{RXR} \gamma$, VDR, TR, LXR $\alpha$ and LXR $\beta$, are expressed by oligodendrocytes and have been shown to affect oligodendroglial function $[5,6,8,26]$. Lack of TR or LXR $\alpha / \operatorname{LXR} \beta$ results in delayed oligodendroglial differentiation or hypomyelination in the cerebellum, respectively $[8,26]$. Similarly, blocking and/or downregulation of VDR, TRs or RXRY impaired oligodendroglial differentiation [5, 27]. In contrast, our results provide no indication that lack of FXR delays oligodendroglial differentiation in vitro or in vivo. FXR forms a heterodimer with RXR to induce expression of target genes, but FXR can also function as a repressor by suppressing gene expression [13]. Our results suggest that, at least in oligodendrocytes, the function of FXR is redundant to or is compensated by other nuclear receptors.

Activation of several nuclear receptors such as RXR $\gamma$, TR, VDR and LXR $\alpha / L X R \beta$ promotes oligodendroglial differentiation [3, 5-8]. This is in contrast to our results, which show that activation of FXR by the synthetic agonist GW4064 had no effect on the number of MBP+ mature oligodendrocytes or expression levels of MBP. Oligodendrocytes display a region-dependent heterogeneity within the CNS [28], and interestingly, lack of $\mathrm{LXR} \alpha / \beta$ is associated with hypomyelination in the cerebellum [8]. To determine whether FXR might play a more prominent role in the differentiation of cerebellar oligodendrocytes, we repeated our in vitro experiments using oligodendrocytes isolated specifically from the cerebellum. However, neither lack nor activation of FXR modulated the differentiation of cerebellar oligodendrocytes. Likewise, addition of FXR agonists to demyelinated cerebellar slice cultures did not exhibit an effect on remyelination in this ex vivo model in which exclusively CNS-resident cells are present.

It has recently been shown that activation of FXR in BMM induced an anti-inflammatory phenotype characterized by downregulation of pro-inflammatory and induction of anti-inflammatory genes [25]. In light of the finding by Miron and colleagues who demonstrated an increased differentiation of rodent oligodendrocytes cultured in the presence of supernatants derived from anti-inflammatory microglial cells, we hypothesized that FXR-activated BMM might indirectly modulate oligodendrocyte differentiation [29]. However, our results provide no evidence that activation of FXR in macrophages has a similar, indirect effect on oligodendroglial differentiation. Oligodendroglial differentiation and remyelination can also be accelerated in vivo by improved clearance of myelin debris by macrophages as it has been shown for the RXR agonist bexarotene [30]. Whether activation of FXR in macrophages may potentially result in increased myelin phagocytosis is currently unknown. However, this could indirectly contribute to oligodendrocyte differentiation and remyelination. 


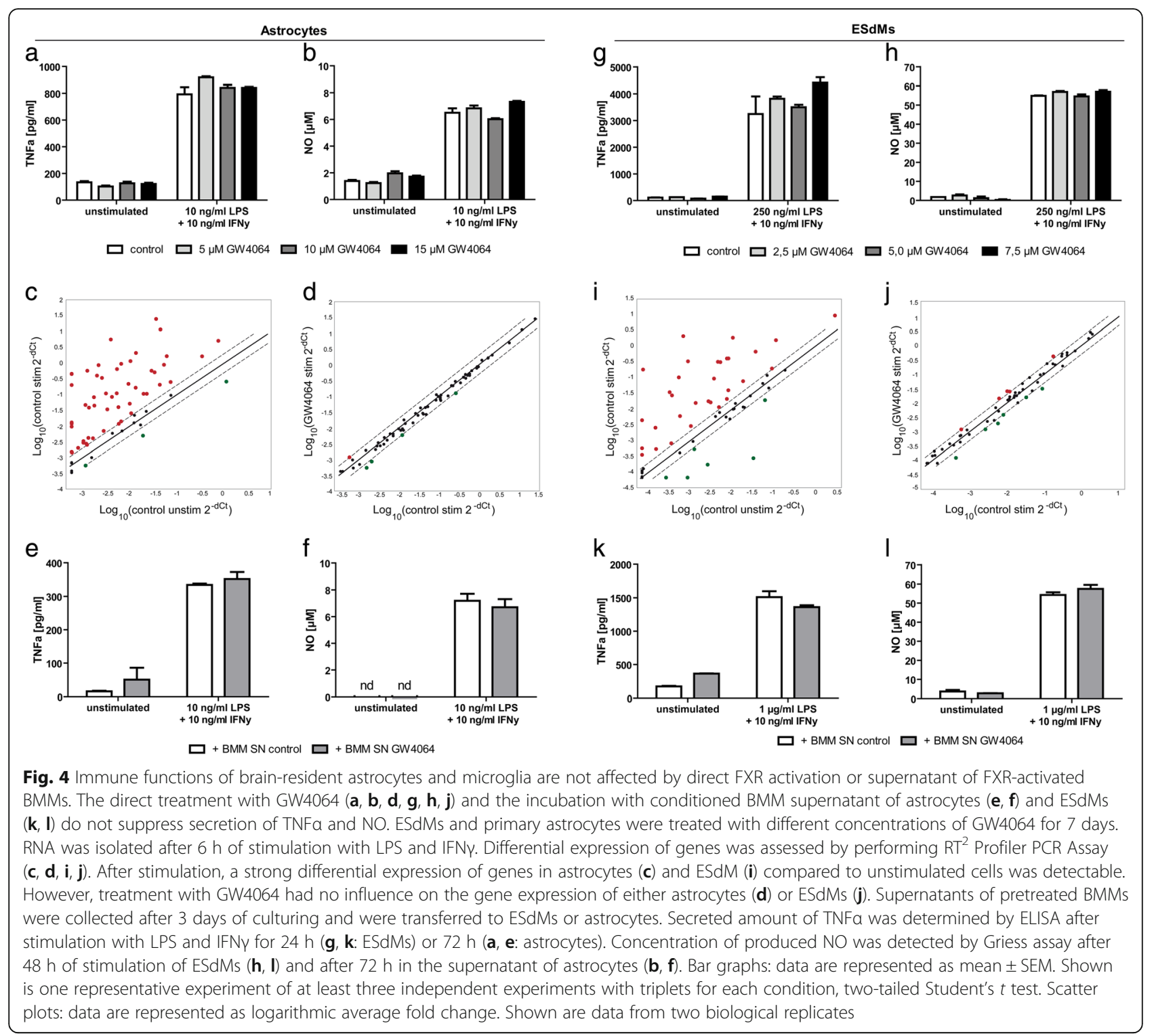

The anti-inflammatory phenotype induced by GW4064mediated activation of FXR in peripheral myeloid cells, such as BMMs, is well documented [15, 25]. However, expression of FXR is not limited to myeloid cells, but is also present in microglia as well as astrocytes [25]. Interestingly, FXR activation in microglia using GW4064 did not result in downregulation of pro-inflammatory key molecules, such as TNFa and NO, or altered expression of pro- and anti-inflammatory genes encoding for cytokines, interleukins or chemokines as well as their receptors. These data illustrate that activation of FXR via GW4064 has no influence on the immune responses of microglia and astrocytes. This contrasts with the activation of other nuclear receptors such as LXR, RXR and PPAR $\alpha[31,32]$. Considering previously published data [25], our results suggest that FXR plays a pivotal role in downregulation of pro-inflammatory molecules in peripheral myeloid cells, but not in CNS-resident microglia suggesting heterogeneity between the two cell populations despite overlapping functions. This may be due to a considerable diversity of gene expression patterns between tissue macrophages from different mouse organs [33] and could further explain the recent observation that blood-derived monocytes might mainly initiate demyelination in EAE whereas microglia potentially primarily clear myelin debris [34].

\section{Conclusion}

In summary, we demonstrated that activation of FXR by GW4064 does not modulate oligodendroglial differentiation directly or indirectly via FXR activation in macrophages. Furthermore, there is no indication that GW4064 directly or indirectly influences the pro- 
inflammatory profiles of CNS-resident astrocytes or microglia. These data suggest that FXR agonists, such as GW4064, represent a potential therapeutic approach for MS which specifically targets peripheral immune cells including macrophages, but not brain-resident cells, such as oligodendrocytes, astrocytes or microglia.

\section{Additional file}

Additional file 1: Figure S1. Cerebellar oligodendroglial differentiation is not affected by direct FXR activation or supernatant of FXR-activated BMMs. Addition of GW4064 during the differentiation of cerebellar oligodendrocytes over 24 and $48 \mathrm{~h}$ does not influence Mbp expression levels (a) or the percentages of PDGFRa+ OPCs or MBP+ oligodendrocytes (b, c). Oligodendrocytes of cerebellar origin exhibit no significant difference in their Mbp expression level when incubated with supernatants from BMM cultured either in the presence or absence of GW4064 (d). The percentage of OPCs is unchanged after incubation with BMM-conditioned medium; the percentage of $\mathrm{MBP}+$ oligodendrocytes is reduced independent of additional GW4064 treatment (e, f). Activation of FXR cultures using 10 and $20 \mu \mathrm{M}$ GW4064 during 14 days of remyelination in cerebellar slice after toxic demyelination does not alter the ratio of $\mathrm{MBP}+$ and $\mathrm{NFL}+$ axonal fibres (g). Remyelinated fibres are exemplarily highlighted (arrows). Note that a high amount of MBP+ debris is still present after 14 days of remyelination (h). In vitro: $n=3$, 1 way ANOVA with Bonferroni's correction, 200 cells per condition were evaluated, ${ }^{*} p<0.05$; ex vivo: $n=2,1$ way ANOVA with Bonferroni's correction, 6 slices with 3 images each per condition and preparation; all images are representative. (TIF $5564 \mathrm{~kb}$ )

\section{Abbreviations}

ANOVA: Analysis of variance; BMM: Bone marrow-derived macrophage; CNS: Central nervous system; CNTF: Ciliary neurotrophic factor; DAPI: 4,6Diamidin-2-phenylindol; DMSO: Dimethylsulfoxid; EAE: Experimental autoimmune encephalomyelitis; ELISA: Enzyme-linked immunosorbent assay; ESdMs: Embryonic stem cell-derived microglial precursor cells; fw: Forward; FXR: Farnesoid-X-receptor; Hsp90ab1: Heat shock protein 90 kDa alpha family class B member 1; ICC: Immunocytochemistry; ICH: Immunohistochemistry; IFNY: Interferon gamma; Ko: Knock out; LPS: Lipopolysaccharide; LSM: Laser scanning microscope: LXR: Liver X receptor; MBP: Myelin basic protein: mRNA: Messenger RNA; MS: Multiple sclerosis; NFL: Neurofilament 70 kDa; NO: Nitric oxide; NogoA: Neurite outgrowth inhibitor A; NR1H4: Farnesoid -X-receptor; NT3: Neurotrophin-3; OL: Mature oligodendrocyte; Olig2: Oligodendrocyte transcription factor; OPC: Oligodendroglial progenitor cell; PDGF: Platelet-derived growth factor; PDGFRa: Platelet-derived growth factor receptor alpha; PFA: Paraformaldehyde; PPAR: Peroxisome proliferatoractivated receptor; RRT-PCR: Quantitative real-time polymerase chain reaction; rev: Reverse; RPLPO: 60S acidic ribosomal protein PO; RXR: Retinoid X receptor; SEM: Standard error of the mean; T3: Triiodothyronine; TNFa: Tumor necrosis factor alpha; TR: Thyroid receptor; VDR: Vitamin D receptor; WT: Wild type

\section{Acknowledgements}

The authors thank Harald Neumann (University of Bonn) for providing the murine embryonic stem cell-derived microglial precursor cells (ESdMs). Additionally, the authors thank Elke Hoffmann and Claudia Kemming for excellent technical assistance.

\section{Funding}

This study was supported by the German Research Foundation (CRC 128 A8 and SFB1009 A03 to LK, CRC 128 B7 and KU1477 6-1 to TK) and the Interdisciplinary Clinical Research Center, Münster (KI2/015/14 to LK and KuT3/012/15).

\section{Availability of data and materials}

The datasets supporting the conclusions of this article are included within the article.

\section{Authors' contributions}

LK and TK designed the study. SA, AKF, SH, IK and ML performed the in vitro and ex vivo experiments. TK analysed mouse tissue samples. SA, AKF, IK and
ML analysed the data. SA, AKF, LK and TK wrote the manuscript. All authors read and approved the final manuscript.

\section{Competing interests}

The authors declare that they have no competing interests.

Consent for publication

Not applicable.

\section{Ethics approval}

Not applicable.

\section{Publisher's Note}

Springer Nature remains neutral with regard to jurisdictional claims in published maps and institutional affiliations.

Received: 26 October 2016 Accepted: 6 March 2017

Published online: 28 March 2017

\section{References}

1. Francis GA, Fayard E, Picard F, Auwerx J. Nuclear receptors and the control of metabolism. Annu Rev Physiol. 2003:65:261-311.

2. Hucke S, Flossdorf J, Grutzke B, Dunay IR, Frenzel K, Jungverdorben J, Linnartz B, Mack M, Peitz M, Brustle O, et al. Licensing of myeloid cells promotes central nervous system autoimmunity and is controlled by peroxisome proliferatoractivated receptor gamma. Brain. 2012;135:1586-605.

3. Chandraratna RA, Noelle RJ, Nowak EC. Treatment with retinoid X receptor agonist IRX4204 ameliorates experimental autoimmune encephalomyelitis. Am J Transl Res. 2016:8:1016-26.

4. Diab A, Hussain RZ, Lovett-Racke AE, Chavis JA, Drew PD, Racke MK. Ligands for the peroxisome proliferator-activated receptor-gamma and the retinoid $X$ receptor exert additive anti-inflammatory effects on experimental autoimmune encephalomyelitis. J Neuroimmunol. 2004;148:116-26.

5. Huang JK, Jarjour AA, Nait Oumesmar B, Kerninon C, Williams A, Krezel W, Kagechika H, Bauer J, Zhao C, Baron-Van Evercooren A, et al. Retinoid X receptor gamma signaling accelerates CNS remyelination. Nat Neurosci. 2011;14:45-53.

6. de la Fuente AG, Errea O, van Wijngaarden P, Gonzalez GA, Kerninon C, Jarjour AA, Lewis HJ, Jones CA, Nait-Oumesmar B, Zhao C, et al. Vitamin D receptorretinoid $\mathrm{X}$ receptor heterodimer signaling regulates oligodendrocyte progenitor cell differentiation. J Cell Biol. 2015;211:975-85.

7. Lee JY, Petratos S. Thyroid hormone signaling in oligodendrocytes: from extracellular transport to intracellular signal. Mol Neurobiol. 2016:53:6568-83.

8. Meffre D, Shackleford G, Hichor M, Gorgievski V, Tzavara ET, Trousson A, Ghoumari AM, Deboux C, Nait Oumesmar B, Liere P, et al. Liver X receptors alpha and beta promote myelination and remyelination in the cerebellum. Proc Natl Acad Sci U S A. 2015;112:7587-92.

9. Lefebvre P, Cariou B, Lien F, Kuipers F, Staels B. Role of bile acids and bile acid receptors in metabolic regulation. Physiol Rev. 2009:89:147-91.

10. Schote AB, Turner JD, Schiltz J, Muller CP. Nuclear receptors in human immune cells: expression and correlations. Mol Immunol. 2007;44:1436-45.

11. Modica S, Gadaleta RM, Moschetta A. Deciphering the nuclear bile acid receptor FXR paradigm. Nucl Recept Signal. 2010;8, e005.

12. Calkin AC, Tontonoz P. Transcriptional integration of metabolism by the nuclear sterol-activated receptors LXR and FXR. Nat Rev Mol Cell Biol. 2012; 13:213-24.

13. Goodwin B, Jones SA, Price RR, Watson MA, McKee DD, Moore LB, Galardi C, Wilson JG, Lewis MC, Roth ME, et al. A regulatory cascade of the nuclear receptors FXR, SHP-1, and LRH-1 represses bile acid biosynthesis. Mol Cell. 2000;6:517-26.

14. Serbina NV, Pamer EG. Monocyte emigration from bone marrow during bacterial infection requires signals mediated by chemokine receptor CCR2. Nat Immunol. 2006:7:311-7.

15. Ho PP, Steinman L. Obeticholic acid, a synthetic bile acid agonist of the farnesoid $X$ receptor, attenuates experimental autoimmune encephalomyelitis. Proc Natl Acad Sci U S A. 2016;113:1600-5.

16. Chang A, Tourtellotte WW, Rudick R, Trapp BD. Premyelinating oligodendrocytes in chronic lesions of multiple sclerosis. New Engl J Med. 2002;346:165-73. 
17. Wolswijk G. Chronic stage multiple sclerosis lesions contain a relatively quiescent population of oligodendrocyte precursor cells. J Neurosci. 1998; 18:601-9.

18. Kuhlmann T, Miron V, Cui Q, Wegner C, Antel J, Brück W. Differentiation block of oligodendroglial progenitor cells as a cause for remyelination failure in chronic multiple sclerosis. Brain. 2008;131:1749-58.

19. Irvine KA, Blakemore WF. Remyelination protects axons from demyelinationassociated axon degeneration. Brain. 2008;131:1464-77.

20. Duncan ID, Brower A, Kondo Y, Curlee Jr JF, Schultz RD. Extensive remyelination of the CNS leads to functional recovery. Proc Natl Acad Sci U S A. 2009;106:6832-6.

21. Sinal CJ, Tohkin M, Miyata M, Ward JM, Lambert G, Gonzalez FJ. Targeted disruption of the nuclear receptor FXR/BAR impairs bile acid and lipid homeostasis. Cell. 2000;102:731-44.

22. Watkins TA, Emery B, Mulinyawe $S$, Barres BA. Distinct stages of myelination regulated by $\gamma$-secretase and astrocytes in a rapidly myelinating CNS coculture system. Neuron. 2009:60:555-69.

23. Preisner A, Albrecht S, Cui Q-L, Hucke S, Ghelman J, Hartmann C, Taketo MM, Antel J, Klotz L, Kuhlmann T. Non-steroidal anti-inflammatory drug indometacin enhances endogenous remyelination. Acta Neuropathol. 2015; 130:247-61.

24. Beutner C, Roy K, Linnartz B, Napoli I, Neumann H. Generation of microglial cells from mouse embryonic stem cells. Nat Protoc. 2010;5:1481-94.

25. Hucke S, Herold M, Liebmann M, Freise N, Lindner M, Fleck AK, Zenker S, Thiebes S, Fernandez-Orth J, Buck D, et al. The farnesoid-X-receptor in myeloid cells controls CNS autoimmunity in an IL-10-dependent fashion. Acta Neuropathol. 2016;132:413-31.

26. Billon N, Jolicoeur C, Tokumoto Y, Vennstrom B, Raff M. Normal timing of oligodendrocyte development depends on thyroid hormone receptor alpha 1 (TRalpha1). EMBO J. 2002;21:6452-60.

27. Huang C, Sakry D, Menzel L, Dangel L, Sebastiani A, Kramer T, Karram K, Engelhard K, Trotter J, Schafer MK. Lack of NG2 exacerbates neurological outcome and modulates glial responses after traumatic brain injury. Glia. 2016;64:507-23.

28. Marques S, Zeisel A, Codeluppi S, van Bruggen D, Mendanha Falcao A, Xiao L, Li H, Haring M, Hochgerner H, Romanov RA, et al. Oligodendrocyte heterogeneity in the mouse juvenile and adult central nervous system. Science. 2016;352:1326-9.

29. Miron VE, Boyd A, Zhao JW, Yuen TJ, Ruckh JM, Shadrach JL, van Wijngaarden P, Wagers AJ, Williams A, Franklin RJ, Ffrench-Constant C. M2 microglia and macrophages drive oligodendrocyte differentiation during CNS remyelination. Nat Neurosci. 2013;16:1211-8.

30. Natrajan MS, de la Fuente AG, Crawford AH, Linehan E, Nunez V, Johnson KR, Wu T, Fitzgerald DC, Ricote M, Bielekova B, Franklin RJ. Retinoid X receptor activation reverses age-related deficiencies in myelin debris phagocytosis and remyelination. Brain. 2015;138:3581-97.

31. Zhang-Gandhi CX, Drew PD. Liver $X$ receptor and retinoid $X$ receptor agonists inhibit inflammatory responses of microglia and astrocytes. J Neuroimmunol. 2007;183:50-9

32. Xu J, Storer PD, Chavis JA, Racke MK, Drew PD. Agonists for the peroxisome proliferator-activated receptor-alpha and the retinoid $X$ receptor inhibit inflammatory responses of microglia. J Neurosci Res. 2005;81:403-11.

33. Gautier EL, Shay T, Miller J, Greter M, Jakubzick C, Ivanov S, Helft J, Chow A, Elpek KG, Gordonov S, et al. Gene-expression profiles and transcriptional regulatory pathways that underlie the identity and diversity of mouse tissue macrophages. Nat Immunol. 2012;13:1118-28.

34. Yamasaki R, Lu H, Butovsky O, Ohno N, Rietsch AM, Cialic R, Wu PM, Doykan CE, Lin J, Cotleur AC, et al. Differential roles of microglia and monocytes in the inflamed central nervous system. J Exp Med. 2014:211:1533-49.

\section{Submit your next manuscript to BioMed Central and we will help you at every step:}

- We accept pre-submission inquiries

- Our selector tool helps you to find the most relevant journal

- We provide round the clock customer support

- Convenient online submission

- Thorough peer review

- Inclusion in PubMed and all major indexing services

- Maximum visibility for your research

Submit your manuscript at www biomedcentral com/submit
C) Biomed Central 